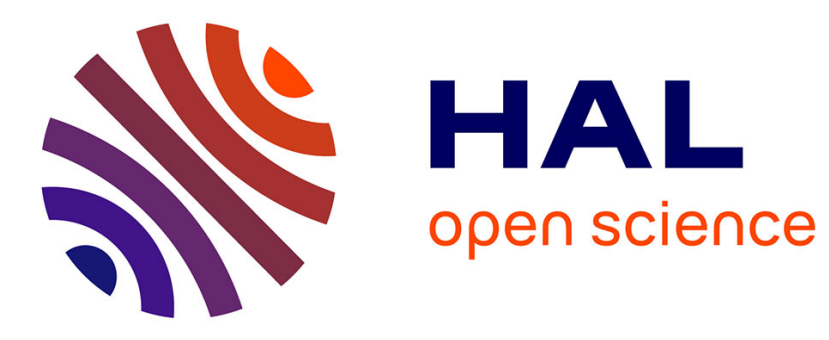

\title{
2-Chloromalonaldehyde, a model system of resonance-assisted hydrogen bonding: vibrational investigation
}

\author{
Michèle Chevalier, Rasa Platakyte, Justinas Ceponkus, Germán \\ Rojas-Lorenzo, Claudine Crépin, Alejandro Gutiérrez-Quintanilla
}

\section{To cite this version:}

Michèle Chevalier, Rasa Platakyte, Justinas Ceponkus, Germán Rojas-Lorenzo, Claudine Crépin, et al.. 2-Chloromalonaldehyde, a model system of resonance-assisted hydrogen bonding: vibrational investigation. Physical Chemistry Chemical Physics, 2018, 20 (18), pp.12888-12897. 10.1039/C7CP06481K . hal-02329417

\section{HAL Id: hal-02329417 \\ https://hal.science/hal-02329417}

Submitted on 1 Feb 2021

HAL is a multi-disciplinary open access archive for the deposit and dissemination of scientific research documents, whether they are published or not. The documents may come from teaching and research institutions in France or abroad, or from public or private research centers.
L'archive ouverte pluridisciplinaire HAL, est destinée au dépôt et à la diffusion de documents scientifiques de niveau recherche, publiés ou non, émanant des établissements d'enseignement et de recherche français ou étrangers, des laboratoires publics ou privés. 


\section{Journal Name}

\section{ARTICLE}

\section{2-Chloromalonaldehyde, a Model System of Resonance-Assisted Hydrogen Bonding: Vibrational Investigation}

Received 00th January 20xx, Accepted 00th January 20xx

DOI: $10.1039 / \times 0 \times x 00000 x$

www.rsc.org/

\begin{abstract}
Alejandro Gutiérrez-Quintanilla, ${ }^{a, b}$, Michèle Chevalier $^{a} *$, Rasa Platakyte ${ }^{a, c}$, Justinas Ceponkus ${ }^{c}$, German A. Rojas-Lorenzo ${ }^{\mathrm{b}}$, Claudine Crépin ${ }^{\mathrm{a}}$

The chelated enol isomer of 2-chloromalonaldehyde (2-CIMA) is experimentally characterized for the first time by IR and Raman spectroscopies. The spectra are obtained by trapping the molecule in cryogenic matrices and analyzed with the assistance of theoretical calculations. Experiments were performed in argon, neon and para-hydrogen matrices. The results highlight puzzling matrix effects, beyond site effects, which are interpreted as due to a tunneling splitting of the vibrational levels related to the proton transfer along the internal hydrogen bond (IHB). 2-CIMA is thus one of the very few molecules in which the $\mathrm{H}$ tunneling has been observed in cryogenic matrices. The comparison with its parent molecule (malonaldehyde) shows experimentally and theoretically the weakening of the IHB upon chlorination, with a reduced cooperative effect in the resonance assisted hydrogen bond. In addition, the $\mathrm{Cl}$ substitution induces an important stabilization of two open enol conformers. These two open forms appear in the spectra of as-deposited samples, meaning that, in contrast with other well-studied molecules of the same family ( $\beta$-dialdehydes and $\beta$-diketones), they are present in the gas phase at room temperature.
\end{abstract}

As submitted to PCCP - 16 january 2018

\footnotetext{
Institut des Sciences Moléculaires d'Orsay (ISMO), UMR 8214, CNRS, Univ. ParisSud, Université Paris-Saclay UMR 8214, F-91405 Orsay, France.

${ }^{b}$ Instituto Superior de Tecnologías y Ciencias Aplicadas (InSTEC), Universidad de La Habana. Ave. Salvador Allende No. 1110, Quinta de los Molinos, La Habana 10400, Cuba.

${ }^{c}$ Department of General Physics and Spectroscopy, Vilnius University, Sauletekio av. 9 bat. III, L-10222 Vilnius, Lithuania.

Email : michele.chevalier@u-psud.fr
}

Electronic Supplementary Information (ESI) available: Theoretical methods and Table of CCC wavenumbers including the whole set of theoretical predictions. See DOI: $10.1039 / \times 0 \times x 00000 x$ 


\section{Introduction}

The hydrogen bond is one of the most important interactions in nature. It is present in inorganic and organic molecules and complexes, playing an important role in the chemistry of life, e.g. water clusters, DNA double helix, etc. Different kinds of hydrogen bonds have been identified according to the classification made by Gilli et al.. ${ }^{1,2}$ The Resonance Assisted Hydrogen Bond (RAHB) is one of the strongest due to the enhancing produced by the presence of some degree of conjugation (for example hydrogen bonding networks in proteins) but there is still some debate on the nature of the interactions at play. ${ }^{3-5}$ The RAHB forms between molecules (intermolecular) and also inside the single molecule as internal hydrogen bonds (IHB, intramolecular) and influences the molecular structure.

Two similar families of molecules have been used as model systems in the studies of intramolecular RAHB systems: malonaldehyde and acetylacetone derivatives. For all these molecules, the two tautomers, the enol form and dialdehyde or diketone forms (keto form) coexist, but RAHB occurs only in the enol form which is the most stable tautomer in the gas phase. Cis enol malonaldehyde (also called CCC form), depicted in Figure $1 \mathrm{a}$, is the simplest molecule with an IHB in a pseudo aromatic ring system allowing a RAHB. It explains the numerous and especially theoretical studies on this molecule. Most of the works focus on the proton transfer. Tunneling of the hydrogen between the two oxygen atoms through the barrier is responsible of the splitting of the energy levels. This splitting is thus an important experimental data related to the $\mathrm{H}$ bond strength. ${ }^{6}$ The tunneling splitting was measured for the ground vibrational state, ${ }^{7-10}$ but it depends on the vibrational level as observed in gas phase ${ }^{11,12}$ or in jet experiments. ${ }^{9,13-15}$ Experimental values of tunnelling splittings for various vibrational modes are quite in agreement with theoretical studies. ${ }^{16-23}$ Surprisingly, no tunnelling was observed for the molecule embedded in matrices ${ }^{15,24-26}$ while it was detected for similar but larger molecules in the same kind of matrices. ${ }^{27,28}$

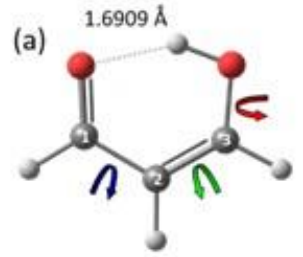

$\mathrm{MA}(\mathrm{CCC})$

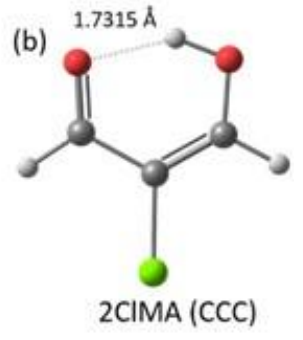

$2 \mathrm{CIMA}(\mathrm{CCC})$
Figure 1: cis enol tautomers ( $\mathrm{CCC}$ ) of (a) malonaldehyde -MA- and (b) 2chloromalonaldehyde -2-CIMA-. The carbon atoms are labelled from one (left) to three (right). The three rotations giving the $X Y Z$ conformers $(X, Y, Z=C$ or $\mathrm{T}$ ) are shown by arrows. The distance between $\mathrm{O}$ and $\mathrm{H}$ in
the IHB is written (theoretical results from this work -see below)
Theoretical calculations predict eight stable enol conformers, called $\mathrm{XYZ}$ with $\mathrm{X}, \mathrm{Y}, \mathrm{Z}=\mathrm{C}$ for cis or $\mathrm{T}$ for trans, obtained by rotation of $180^{\circ}$ around the $C_{1}-C_{2}, C_{2}$ $\mathrm{C}_{3}$ or/and $\mathrm{C}_{3}-\mathrm{O}$ bond respectively (see labels in Figure 1 ). Only the CCC exhibits an IHB. The energy shift between CCC and CCT forms gives an idea of the strength of the $\mathrm{IHB}^{29}$

The substitution of both 'external' $\mathrm{H}$ (attached to $\mathrm{C}_{1}$ and $C_{3}$ ) by methyl groups leads to acetylacetone (ACAC). There are also numerous theoretical and experimental studies on this molecule. ${ }^{30-37}$ Again, the CCC form is stabilized by RAHB. The barrier to $\mathrm{H}$ transfer is slightly smaller than in malonaldehyde $\mathrm{e}^{33,38}$ but the major difference is that the transfer is coupled with internal rotations of the methyl groups ${ }^{37}$. Halogenated derivatives of acetylacetone have also been studied to investigate the influence of the substituent on the $\mathrm{H}$ bond strength. ${ }^{39-42}$ The comparison between chloroacetylacetone (3-chloro-pentane-2,4-dione, $\mathrm{CIACAc)}$ and acetylacetone is of special interest for us. A stronger IHB was found in the chlorinated derivative, explained by a steric effect. ${ }^{41,42}$

Whereas malonaldehyde is a much simpler case than acetylacetone the influence of different substituents to the $\mathrm{H}$ atoms in its photodynamics as well as in its hydrogen bond strength have been investigated only in a few studies. ${ }^{29,43-47}$. In particular, little information is available on 2-chloromalonaldehyde (noted hereafter 2CIMA). Compare to malonaldehyde (noted hereafter $M A)$, this chlorinated derivative, depicted in Figure $1 \mathrm{~b}$, shows a very similar RAHB with symmetric potential wells in the $\mathrm{H}$ transfer. To our knowledge, only theoretical works are published about the structure of this molecule. They concern mainly theoretical calculations on the CCC form with a focus on the IHB. ${ }^{29,43,44,47,48}$ The geometry, the barrier height for $\mathrm{H}$ transfer and the associated splitting in the ground state were calculated for different derivatives of MA obtained by the substitution of one of the $\mathrm{H}$ atoms in different positions. Rios et al. ${ }^{44}$ obtained an estimation of the ground state splitting of $22 \mathrm{~cm}^{-1}$ for MA, in good agreement with experimental data, ${ }^{8,10}$ and of about 12 $\mathrm{cm}^{-1}$ for 2-CIMA. The chlorine has different effects depending on its position: (i) electron withdrawing due to its electronegativity, (ii) electron donor through its lone pairs, one lone pair can participate to conjugation of the $\pi$ system or can make a $\mathrm{H}$ bond (IHB or external), and (iii) steric effect on the neighbors because of the large size of $\mathrm{Cl}$ compared to $\mathrm{H}$. The substitution of a chlorine atom on the $\alpha$-position $\left(C_{2}\right.$, see Figure 1$)$ does not enhance the conjugation effect. The distance between chlorine and the closer $\mathrm{H}$ atom in the CCC conformer is large enough to avoid an IHB between them. All these theoretical studies predict that for an isolated molecule, chlorination in $\alpha$-position should weaken the $\mathrm{H}$ bond.

This paper presents the first spectroscopic study of 2CIMA, allowing to check this assumption. The vibrational spectroscopy gives access to an investigation 
of the influence of the IHB on the structure and dynamics of $\mathrm{H}$ transfer in 2-CIMA. IR and Raman spectroscopies were combined to collect a large set of vibrational data. Trapping in different cryogenic matrices was achieved: para-hydrogen $\left(p \mathrm{H}_{2}\right)$ matrix, a soft solid in which large amplitude motions of a guest are preserved, ${ }^{49}$ neon matrix, the least perturbing matrix in terms of vibrational frequencies compared to gas phase values and argon matrix, a more rigid solid with different sizes for the guest sites widely used in matrix isolation spectroscopy.

The $\mathrm{H}$ transfer in the $\mathrm{CCC}$ form can be more or less hindered depending on these environments. In addition, quantum chemical calculations were performed to describe the structure of the molecule, especially in comparison with $\mathrm{MA}$, and to obtain valuable predictions on vibrational frequencies, IR intensities and Raman activities of all the vibrational modes of CCC. Theoretical investigations were performed using the density functional theory (DFT) approach, with the M06-2X functional and 6$311++\mathrm{G}(3 \mathrm{df}, 3 \mathrm{dp})$ basis set. The experimental and theoretical methods are detailed in the Supporting Information (ESI).

\section{Results}

\section{Theoretical calculations}

The geometry obtained for the CCC cis-enol of 2-CIMA is of $C_{\mathrm{s}}$ symmetry (Figure $1 \mathrm{~b}$ ). The geometrical parameters are summarized and compared to those of MA in Table 1. The insertion of the bulky chlorine in $\alpha$ position does not modify the structure of the carbonated skeleton, which maintains its planarity. The slight differences in angles and bond distances are consistent with the expected slight decrease of the IHB in 2-CIMA. The most important change, except $\mathrm{C}-\mathrm{Cl}$ distance, is effectively manifested in the $-\mathrm{O}-\mathrm{H} \cdots \mathrm{O}$ geometry $(d(\mathrm{O} \cdots \mathrm{H})$ is indicated in Figure 1). One can notice that our results are in good agreement with previous theoretical works (see Tables 1 and 2 in ref. 29) and very close to the structure deduced from experiments in the case of MA, ${ }^{7}$ included in Table 1 for comparison.

The energies of the enol conformers of 2-CIMA and MA were calculated. A more detailed description of the results will be given in a forthcoming publication dedicated to the open enol conformers of 2-CIMA. ${ }^{50}$ CCC is found to be the most stable conformer, as expected, but two open enol forms appear to have only slightly higher energies in the case of 2-CIMA: TTC and CTC, at $7.0 \mathrm{~kJ} \cdot \mathrm{mol}^{-1}$ and $11.3 \mathrm{~kJ} \cdot \mathrm{mol}^{-1}$ above CCC, respectively. Of interest are the relative energies of CCC and CCT conformers, corresponding to the opening of the IHB. The Zero-Point Energy corrected theoretical energies for CCT, related to CCC are $45.4 \mathrm{~kJ}^{\mathrm{mol}}{ }^{-1}$ for 2$\mathrm{CIMA}$ and $50.5 \mathrm{~kJ} \mathrm{~mol}^{-1}$ for MA, indicating a weakening of the IHB upon chlorination.
Table 1: Geometries of MA and 2-CIMA in their enol CCC forms ( $C_{s}$ symmetry), obtained in the present theoretical M06-2X/6$311++G(3 d f, 3 p d)$ calculations, compared with experimental data in the case of MA. ${ }^{7}$ Distances $(R)$ in $\AA$, angles $(A)$ in degrees. $X=H / C l$ in $M A / 2$ CIMA respectively. Numbering of $C$ atoms is shown in Figure 1.

\begin{tabular}{|c|c|c|c|}
\hline & \multicolumn{2}{|l|}{ MA } & \multirow{2}{*}{$\begin{array}{l}\text { 2-ClMA } \\
\text { Calc. }\end{array}$} \\
\hline & Exp. $^{7}$ & Calc. & \\
\hline $\mathrm{R}\left(\mathrm{C}_{3}-\mathrm{H}\right)$ & 1.089 & 1.084 & 1.083 \\
\hline $\mathrm{R}\left(\mathrm{C}_{2}-\mathrm{C}_{3}\right)$ & 1.348 & 1.355 & 1.352 \\
\hline $\mathrm{R}\left(\mathrm{C}_{3}-\mathrm{O}\right)$ & 1.320 & 1.312 & 1.314 \\
\hline $\mathrm{R}\left(\mathrm{C}_{1}-\mathrm{C}_{2}\right)$ & 1.454 & 1.440 & 1.448 \\
\hline $\mathrm{R}\left(\mathrm{C}_{2}-\mathrm{X}\right)$ & 1.091 & 1.077 & 1.727 \\
\hline $\mathrm{R}\left(\mathrm{C}_{1}-\mathrm{H}\right)$ & 1.094 & 1.099 & 1.099 \\
\hline $\mathrm{R}\left(\mathrm{C}_{1}-\mathrm{O}\right)$ & 1.234 & 1.226 & 1.220 \\
\hline $\mathrm{R}(\mathrm{O}-\mathrm{H})$ & 0.969 & 0.990 & 0.985 \\
\hline $\mathrm{R}(\mathrm{O} \cdots \mathrm{H})$ & 1.68 & 1.691 & 1.732 \\
\hline $\mathrm{A}\left(\mathrm{H}-\mathrm{C}_{3}-\mathrm{C}_{2}\right)$ & 122.3 & 122.2 & 121.6 \\
\hline $\mathrm{A}\left(\mathrm{H}-\mathrm{C}_{3}-\mathrm{O}\right)$ & & 113.5 & 114.4 \\
\hline $\mathrm{A}\left(\mathrm{C}_{2}-\mathrm{C}_{3}-\mathrm{O}\right)$ & 124.5 & 124.4 & 124.0 \\
\hline $\mathrm{A}\left(\mathrm{C}_{3}-\mathrm{C}_{2}-\mathrm{C}_{1}\right)$ & 119.4 & 119.3 & 120.7 \\
\hline $\mathrm{A}\left(\mathrm{C}_{3}-\mathrm{C}_{2}-\mathrm{X}\right)$ & 128.1 & 120.2 & 120.8 \\
\hline $\mathrm{A}\left(\mathrm{C}_{3}-\mathrm{O}-\mathrm{H}\right)$ & 106.3 & 106.8 & 107.7 \\
\hline $\mathrm{A}\left(\mathrm{C}_{1}-\mathrm{C}_{2}-\mathrm{X}\right)$ & & 120.5 & 118.5 \\
\hline $\mathrm{A}\left(\mathrm{C}_{2}-\mathrm{C}_{1}-\mathrm{H}\right)$ & 117.6 & 117.4 & 116.7 \\
\hline $\mathrm{A}\left(\mathrm{C}_{2}-\mathrm{C}_{1}-\mathrm{O}\right)$ & 123.0 & 123.5 & 122.5 \\
\hline $\mathrm{A}\left(\mathrm{H}-\mathrm{C}_{1}-\mathrm{O}\right)$ & & 119.1 & 120.8 \\
\hline $\mathrm{A}\left(\mathrm{C}_{1}-\mathrm{O} \cdots \mathrm{H}\right)$ & & 100.4 & 101.0 \\
\hline $\mathrm{A}(\mathrm{O}-\mathrm{H} \cdots \mathrm{O})$ & 147.6 & 145.7 & 144.0 \\
\hline
\end{tabular}

The whole description of the vibrational modes (wavenumbers, IR intensities, Raman intensities) obtained in the present calculations is reported in ESI, Table S1. The relevant values for comparison with experiments are given in Table 2.

\section{Experiments}

\section{Infrared spectroscopy of as-deposited samples}

The IR spectra in the $700-1800 \mathrm{~cm}^{-1}$ region of 2-CIMA in argon, para-hydrogen and neon matrices are shown in Figure 2 (panels a, b and c respectively). The calculated spectrum for the CCC conformer is also shown in panel (d). The spectra in the different matrices are similar and the theoretical prediction of the CCC form is in good agreement with them. This indicates that the CCC conformer prevails as main structure, as expected from the energy calculations, and that the assignment of the CCC bands is made with high confidence. Table 2 reports the experimental vibrational frequencies of CCC in the three matrices, together with theoretical values and a short mode description. 


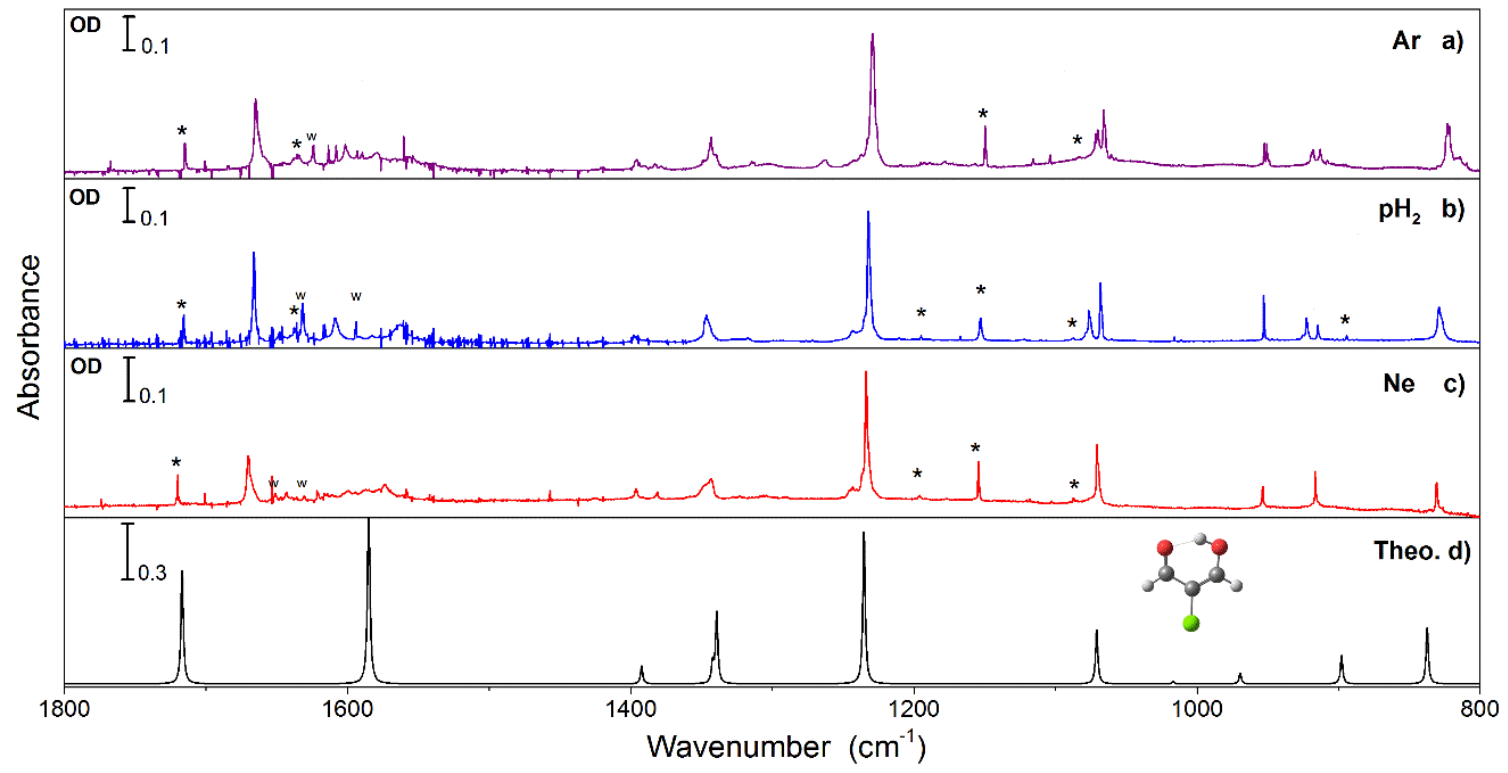

Figure 2: Infrared absorption spectra of as deposited 2-CIMA in (a) $\mathrm{Ar}(7 \mathrm{~K})$, (b) $p \mathrm{H}_{2}(3 \mathrm{~K})$ and (c) Ne (7 K) matrices. (d) Theoretical calculation (arbitrary units) of CCC conformer in harmonic approximation with 0.968 scaling factor, a Lorentzian profile of $\mathrm{FWHM}=2 \mathrm{~cm}^{-1}$ is used; ${ }^{*}$ denoter bands belonging to open
conformers,

One can notice that the $\mathrm{O}-\mathrm{H}$ stretching mode, predicted with a high IR intensity, is not observed because of its huge broadening due to the IHB, similarly to the case of malonaldehyde.

Comparison of experimental and theoretical spectra shows that several bands of weak intensity cannot be assigned to CCC. This is confirmed by UV irradiation experiments in which photoisomerization of CCC is observed. ${ }^{50}$ These experiments combined with theoretical calculations show that the bands marked with an asterisk $\left({ }^{*}\right)$ in Figure 2 belong to the open enol conformer of lowest energy: TTC. As discussed previously, there are two open forms quite low in energy, namely TTC and CTC. The population of these forms at room temperature can be theoretically estimated assuming a thermal equilibrium to about $6 \%$ of TTC and $1 \%$ of CTC in addition of CCC. Using the harmonic intensities of isolated bands (TTC at $1150 \mathrm{~cm}^{-1}$ and CCC at $1232 \mathrm{~cm}^{-1}$ ), we found slightly less than $10 \%$ of TTC in all the matrices, and traces of CTC (about 1\%) are also detected in the region of its most intense band (around $1615 \mathrm{~cm}^{-1}$ ). The presence of TTC and CTC in the matrix is explained by the fast cooling of the gas mixture on the deposition window when preparing the sample, freezing the room temperature thermal equilibrium of conformers. They are stabilised in the matrices and their concentrations do not change with time, as it was proved by the experiment where the matrix after deposition was kept at low temperature for four days. Their observation confirms the order of magnitude of the calculated energies of the enol forms. 2-CIMA is thus the first member of the $\beta$-aldehyde and $\beta$-diketone families showing evidence of stable open enol forms in the gas phase.
Besides the usual frequency shift from one host to another, differences appear between the three experimental spectra. Figure 3 zooms in on two bands in the $1100-900 \mathrm{~cm}^{-1}$ region. These bands are split in doublets in $\mathrm{pH}_{2}$ and in Ar with splitting of $8 \mathrm{~cm}^{-1}$ and 4-5 $\mathrm{cm}^{-1}$ respectively; they are not split in $\mathrm{Ne}$ and correspond to the bands at 1070.6 and $916.5 \mathrm{~cm}^{-1}$ in that matrix. A weak band, redshifted from the intense band around $1345 \mathrm{~cm}^{-1}$, is detected only in $\mathrm{Ar}$ and $\mathrm{pH}_{2}$ (at 1314 and $1317 \mathrm{~cm}^{-1}$ respectively) and could be considered as a low energy component of a doublet. At last, a large splitting is observed on the most intense mode predicted at $1584.9 \mathrm{~cm}^{-1}: 46 \mathrm{~cm}^{-1}$ in $\mathrm{pH}_{2}$ and 22 $\mathrm{cm}^{-1}$ in $\mathrm{Ar}$. In the heavier $\mathrm{Ar}$, the observation of structures in vibrational bands can be due to site effects, but the similarities with the $\mathrm{pH}_{2}$ spectrum suggest another physical effect. In fact, site effects are effectively observed in many bands in $\mathrm{Ar}$ (and less pronounced in $\mathrm{Ne}$ ), without splitting larger than $3 \mathrm{~cm}^{-1}$. In $p \mathrm{H}_{2}$ the site effect doublets are detected when $f c c$ and $h c p$ ordering coexist, ${ }^{51}$ or when the amount of $\mathrm{oH}_{2}$ impurities is large. We annealed the $p \mathrm{H}_{2}$ samples at 4.2 $\mathrm{K}$ and waited at least 2 hours to check the purity of our hcp $p \mathrm{H}_{2}$ samples: no evolution of the spectra appeared thus proving that our $\mathrm{pH}_{2}$ matrix consists only of one type crystal. On the other hand, the lineshapes, the values of the splitting and the intensity ratio did not change with different amounts of water impurities, or guest concentration (which remained always low) excluding the attribution of doublets to complexes. These doublets will be analyzed in the Discussion. 
Table 2: IR band positions $\left(\mathrm{cm}^{-1}\right)$ of $\mathrm{CCC}$ in $\mathrm{Ne}, \mathrm{pH}_{2}$ and $\mathrm{Ar}$ matrices, compared with scaled harmonic calculated wavenumbers. Theoretical IR intensities in $\mathrm{km} / \mathrm{mol}$ and Raman activities in $\AA^{4} / \mathrm{amu}$. Values in italic correspond to bands recorded in the Raman spectrum.

\begin{tabular}{|c|c|c|c|c|c|c|}
\hline \multicolumn{3}{|c|}{ Experimental $^{\mathrm{a}}$} & \multicolumn{4}{|c|}{ Calculations } \\
\hline $\mathrm{Ne}$ & $p \mathrm{H}_{2}$ & $\mathrm{Ar}$ & Mode & $\mathrm{CCC}$ & & \\
\hline \multirow[t]{2}{*}{ IR } & IR & IR/Ram. & $\begin{array}{l}\text { descriptio } \\
n^{\text {b }}\end{array}$ & $\tilde{\mathrm{v}}, \mathrm{cm}^{-1}$ & $\begin{array}{l}\text { IR } \\
\text { int }\end{array}$ & $\begin{array}{l}R . \\
a c t\end{array}$ \\
\hline & & & $v \mathrm{OH}$ & 3280.8 & 208 & 24 \\
\hline 2917 & 2909.8 & 2910 & $v \mathrm{C}_{3} \mathrm{H}$ & 3112.0 & 8 & 82 \\
\hline 2875.1 & 2876.7 & $2885.8 / 2883$ & $v \mathrm{C}_{1} \mathrm{H}$ & 2945.5 & 40 & 96 \\
\hline 1670.2 & 1665.8 & $1664.7 / 1665$ & $\begin{array}{l}(v \mathrm{C}=\mathrm{O}+ \\
v \mathrm{C}=\mathrm{C}) \\
(\text { oph })\end{array}$ & 1716.6 & 175 & 13 \\
\hline $\begin{array}{l}1587- \\
1575\end{array}$ & 1563.0 & $1601.5 / 1598$ & $\begin{array}{l}\delta \mathrm{OH}+ \\
(v \mathrm{C}=\mathrm{O}+ \\
v \mathrm{C}=\mathrm{C}) \\
(\mathrm{iph})^{\mathrm{c}}\end{array}$ & 1584.9 & 303 & 62 \\
\hline 1396.2 & 1397.0 & 1396.2 & $\begin{array}{l}\left(\delta \mathrm{C}_{1} \mathrm{H}+\right. \\
\left.\delta \mathrm{C}_{3} \mathrm{H}\right) \\
(\mathrm{iph})\end{array}$ & 1392.1 & 28 & 1 \\
\hline 1347.7 & 1346.9 & & $\begin{array}{l}\left(\delta \mathrm{C}_{1} \mathrm{H}+\right. \\
\left.\delta \mathrm{C}_{3} \mathrm{H}\right) \\
(\mathrm{oph})\end{array}$ & 1342.1 & 31 & 9 \\
\hline \multirow{2}{*}{1343.0} & 1346 & $1343.4 / 1344$ & \multirow{2}{*}{$\delta \mathrm{OH}^{\mathrm{d}}$} & \multirow{2}{*}{1339.2} & \multirow{2}{*}{110} & \multirow{2}{*}{24} \\
\hline & 1317 & $1314 / 1317$ & & & & \\
\hline 1233.6 & 1232.3 & 1229.0 & $\begin{array}{l}\delta \mathrm{OH}+ \\
\delta \mathrm{C}_{3} \mathrm{H}\end{array}$ & 1235.1 & 236 & 0.1 \\
\hline \multirow[t]{2}{*}{1070.6} & $\begin{array}{l}1076.4 \\
1068.1\end{array}$ & 1070/1068 & $\begin{array}{l}v \mathrm{C}-\mathrm{C}+ \\
\delta \mathrm{CCC}+ \\
v \mathrm{C}-\mathrm{Cl}\end{array}$ & 1070.8 & 83 & 6 \\
\hline & 1016 & $1013 \mid 1010$ & $\begin{array}{l}\left(\gamma \mathrm{C}_{1} \mathrm{H}+\right. \\
\left.\gamma \mathrm{C}_{3} \mathrm{H}\right) \\
(\mathrm{oph})\end{array}$ & 1016.9 & 4 & 0.4 \\
\hline 953.7 & 952.8 & $952.7 \mid 950.8$ & $\begin{array}{l}\left(\gamma \mathrm{C}_{1} \mathrm{H}+\right. \\
\left.\gamma \mathrm{C}_{3} \mathrm{H}\right) \\
\text { (iph) }\end{array}$ & 969.4 & 17 & 1 \\
\hline \multirow{2}{*}{916.5} & 922.5 & 918 & \multirow{2}{*}{$\Delta$} & \multirow{2}{*}{897.8} & \multirow{2}{*}{44} & \multirow{2}{*}{12} \\
\hline & 914.7 & $913.1 / 914$ & & & & \\
\hline \multirow[t]{5}{*}{830.8} & 829.3 & 823.5 & $\gamma \mathrm{OH}$ & 837.4 & 87 & 1 \\
\hline & & 505 & $\Delta$ & 500.6 & 16 & 5 \\
\hline & & 474 & $\Delta+v \mathrm{C}-\mathrm{Cl}$ & 470.7 & 6 & 7 \\
\hline & & 278 & $\delta \mathrm{CCl}$ & 277.9 & 4 & 3 \\
\hline & & 225 & $\gamma \mathrm{C}_{1} \mathrm{H}$ & 212.3 & 6 & 1 \\
\hline
\end{tabular}

${ }^{a}$ site structures reported as value1|value2; ${ }^{b} v$ - stretching, $\delta$-in plane bending, $\gamma$-out of plane bending, $\Delta$-in plane ring deformation, (oph) out of phase, (iph) in phase, $\Gamma$-out of plane ring deformation, labelling of carbon atoms from Figure $1 ;^{\mathrm{c}}$ a resonance with $\delta \mathrm{OH}+$ $\delta \mathrm{CCC}\left(233 \mathrm{~cm}^{-1}\right)$ and ${ }^{\mathrm{d}}$ a resonance with $\left(\gamma \mathrm{C}_{1} \mathrm{H}+\gamma \mathrm{C}_{3} \mathrm{H}\right)$ (iph) $+\Gamma$ $\left(369 \mathrm{~cm}^{-1}\right)$ appear in anharmonic calculations.

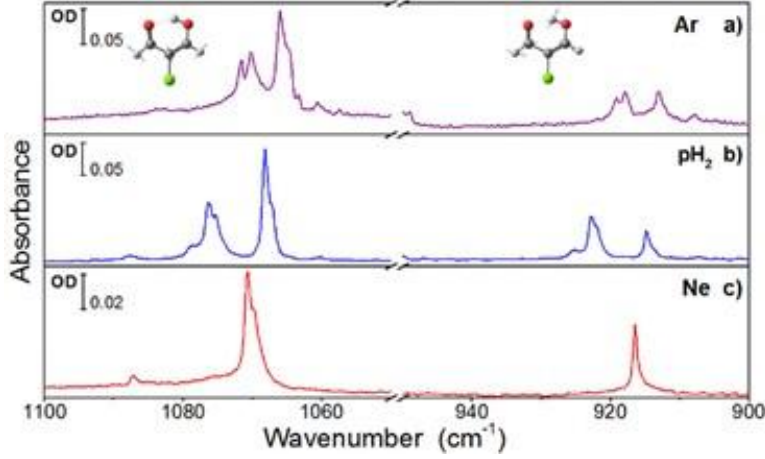

Figure 3: Zoom of the $1100-900 \mathrm{~cm}^{-1}$ spectral region showing the splittings in $\mathrm{Ar}$ (a) and $\mathrm{pH}_{2}$ (b) matrices of the two modes observed at 1070.6 and $916.5 \mathrm{~cm}$ in $\mathrm{Ne}$ (spectrum in panel c). Corresponding normal modes are depicted.

The last remark on the IR spectra concerns the structure of some vibrational bands due to the presence of the two stable isotopes of chlorine atom, ${ }^{35} \mathrm{Cl}$ and ${ }^{37} \mathrm{Cl}$, with $75.78 \%$ and $24.22 \%$ of natural abundance. Theoretical calculations were performed for both isotopologues. The frequency differences are very small and cannot be observed for most of the modes except for those in which the $\mathrm{C}-\mathrm{Cl}$ stretching has a quite high relative weight. The largest difference is expected for the mode predicted at $470.7 \mathrm{~cm}^{-1}$, corresponding to the $\mathrm{C}-\mathrm{Cl}$ stretch, not recorded with our FT-IR setup but observed in Raman (see below). Experimental observable differences have been measured for two other modes as well. These modes correspond to the two doublets previously discussed and shown in Figure 3. When looking carefully, each component of the doublet presents a shoulder in the low wavenumber side, assigned to the ${ }^{37} \mathrm{Cl}$ isotopologue. The shift of $1.1 \mathrm{~cm}^{-1}$ for the mode at $1070.6 \mathrm{~cm}^{-1}$ (in Ne) is in agreement with the theoretical value of $1.2 \mathrm{~cm}^{-1}$. For the mode at 916.5 $\mathrm{cm}^{-1}$ (in Ne) the theoretical value of $0.3 \mathrm{~cm}^{-1}$ is smaller than the experimentally observed at $0.6 \mathrm{~cm}^{-1}$, but is still in the uncertainty error. The ratios between the intensities of each component in those modes are closer to 0.33 , the theoretical expected value.

\section{Raman spectrum in argon matrix}

Raman spectrum was recorded after deposition as a complementary experiment to FT-IR. The spectrum was hard to obtain for this molecule because of its low vapor pressure, even at $80^{\circ} \mathrm{C}$. It is shown in Figure 4 along with the theoretical Raman spectrum of the CCC conformer for comparison. The spectrum covers a more extended range than IR spectra in the low region of wavenumbers. As the selection rules are different, the intensities of the Raman bands differ from those of the IR bands. The Raman spectrum leaves no doubt about the presence of the CCC conformer. The match is good for most of the theoretical normal modes. In the low frequency side, the $\mathrm{C}-\mathrm{Cl}$ stretch at $474 \mathrm{~cm}^{-1}$ is clearly visible. This mode, the most sensible to the isotopic effect, shows two components separated by $6 \mathrm{~cm}^{-1}$ as theoretically predicted. A broad and structured feature 
is observed on the intense mode predicted at 1584.9 $\mathrm{cm}^{-1}$ that exhibits a splitting of $22 \mathrm{~cm}^{-1}$ in IR. This feature is slightly red shifted from the IR values. Due to the higher concentration compared to samples in FT-IR experiments, presence of dimers or clusters in the sample could not be excluded, and could explain this behavior. Only the second component of the 918-913 $\mathrm{cm}^{-1}$ doublet is observed in Raman despite the $2 \mathrm{~cm}^{-1}$ resolution and the band is quite broad. A weak, broad and not well defined band is observed around $1068 \mathrm{~cm}^{-1}$ in the range of the $1066-1070 \mathrm{~cm}^{-1}$ doublet.

As for the IR spectrum, there are some extra bands. We identify the presence of slightly less than $10 \%$ of TTC as in the IR spectrum of the deposited sample.

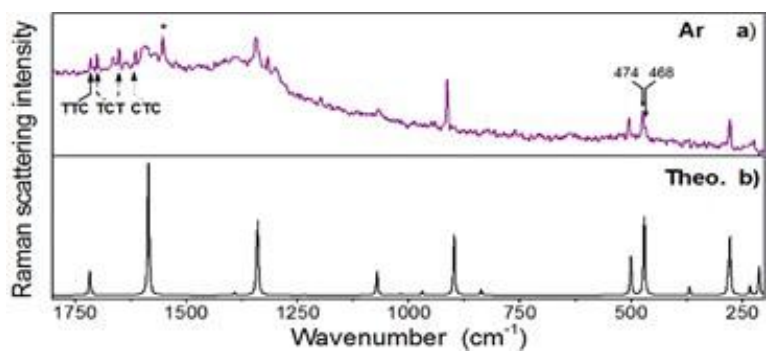

Figure 4: Raman spectra of 2-CIMA in the low frequency region. (a) experimental spectrum in $\mathrm{Ar}$ at $11 \mathrm{~K}$, (b) scaled theoretical harmonic

Surprisingly, the remaining intense bands in the 1600 $1700 \mathrm{~cm}^{-1}$ spectral range could belong to CTC and TCT, giving also an estimation of about $8-10 \%$ of CTC and TCT in the sample. As TCT was not observed and only $1 \%$ of CTC was detected in the IR measurements, we conclude that the laser used for Raman probably induces photoisomerization. The measured Raman frequencies of CCC are reported in italic in Table 2.

\section{Discussion}

The properties of 2-CIMA can be compared to those of its parent molecule (malonaldehyde - MA) and those of the simplest $\beta$-diketone (acetylacetone -AcAc) which have been studied in similar experimental conditions in previous works. ${ }^{26,31,35}$

\section{Strength of IHB}

Various properties can be examined in order to compare the strength of the IHB in a series of molecules: geometrical parameters, conformation energies, experimental spectroscopic parameters or even harmonic frequency calculations. ${ }^{29}$ The question of tunnelling effects, strongly related to the strength of IHB will be discussed in the following part.

Previous calculations have shown that the molecular structure of 2-CIMA corresponds to a weaker IHB than in $\mathrm{MA}$ with a larger $\mathrm{O} \cdots \mathrm{O}$ distance and a smaller $\mathrm{O}-\mathrm{H} \cdots \mathrm{O}$ angle. The presented theoretical results give the same trend (Table 1). These two geometrical parameters are reported in Table 3 for 2-CIMA, MA and AcAc using the same theoretical method (M06-2X/6-311++G(3df,3pd)). A coupling parameter was also introduced by $G$. Gilli and P. Gilli ${ }^{1}$ to quantify the cooperative effect in a RAHB. This parameter, reported in Table 3 , is linked to the geometry of the molecule:

$$
\lambda=\frac{\left(1-\frac{Q}{0.320}\right)}{2}
$$

where $Q=q_{1}+q_{2}=d_{C-O}-d_{C=O}+d_{C-C}-d_{C=C}$ is known as the coordinate of $\pi$ bond delocalization. Values approaching 0.5 indicates higher level of delocalization. All these geometrical parameters show a strength of IHB following 2-CIMA $<\mathrm{MA}<\mathrm{AcAc}$. $\lambda(2-\mathrm{CIMA})$ is significantly lower than $\lambda(M A)$ and $\lambda(A c A c)$, indicating a loss of cooperative effects in 2-CIMA.

Some theoretical estimations of the energy of the $\mathrm{H}$ bond use the energy difference between the stable and the transition states of the $\mathrm{H}$ transfer, or between the cis and trans conformers (CCC and CCT). ${ }^{29,44}$ The latter energy can be extracted from the present calculations showing the same trend as the molecular geometries (Table 3).

There are several spectroscopic evidences of the strength of an IHB. The most obvious one is the broadening and a red shift of the $\mathrm{OH}$ stretching mode. This mode is not observed in matrices for the three molecules because of a too large broadening. Nevertheless, some other bands are broadened, due to the IHB, with a much less expressed broadening, but it is difficult to compare the bandwidths from one molecule to another. Firstly, spectra were obtained with different experimental conditions. The best should be to compare the bandwidths in $p \mathrm{H}_{2}$ matrices, because site effects are minimized in this solid, but there are no available data on MA trapped in $\mathrm{pH}_{2}$. However, it is obvious that many bands of AcAc are much broader than those of 2-CIMA when the molecules are trapped in $\mathrm{pH}_{2}{ }^{35,37}$ especially clear for the intense modes around $1600-1650 \mathrm{~cm}^{-1}$ corresponding to the same kind of modes in both molecules. Secondly, there are two other large amplitude motions in AcAc (torsion of methyl groups): the observed broadening can also come from the coupling with these hydrogen motions. Beside the shift of the $\mathrm{OH}$ stretching mode, one can consider the frequency shifts on the $\mathrm{OH}$ bending modes. Stronger is the IHB, bluer is the frequency shift on these modes. The in-plane bending motion $(\delta \mathrm{OH})$ is delocalized in many modes in the three molecules, depending on the molecule, whereas the out of plane $(\gamma \mathrm{OH})$ bending mode is well defined. The comparison between $\gamma \mathrm{OH}$ values is thus more reliable as a "measure" of the strength of the IHB. Values of $\mathrm{\gamma OH}$ measured in $\mathrm{Ne}$ matrices for the three molecules are reported in Table 3. All these data give the same trend. The IHB strength decreases from acetylacetone to 2chloromalonaldehyde. The experimental data confirm the theoretical studies. An electronegative atom in $\alpha$-position decreases the delocalization on the pseudo aromatic ring, weakening the IHB. 
Table 3: Parameters related to the strength of the IHB: $d(O \cdots O)$ distance $[\AA ̊], \mathrm{O}-\mathrm{H} \cdots \mathrm{O}$ angle $\left[{ }^{\circ}\right]$, coupling parameter $(\lambda)$, relative energies of the CCT conformer to $\mathrm{CCC}(\Delta \mathrm{E}[\mathrm{kJ} / \mathrm{mol}])$ and experimental frequencies of $\gamma \mathrm{OH}\left[\mathrm{cm}^{-1}\right]$ in Ne matrix.

\begin{tabular}{llll}
\hline & 2-ClMA & MA & AcAc \\
\hline $\mathrm{d}(\mathrm{O} \cdots \mathrm{O})$ & 2.594 & 2.570 & 2.528 \\
Angle O-H $\cdots \mathrm{O}$ & 144.03 & 145.66 & 148.44 \\
$\lambda$ & 0.203 & 0.232 & 0.237 \\
$\Delta \mathrm{E}(\mathrm{CCT}-\mathrm{CCC})$ & 45 & 51 & 63 \\
$\gamma \mathrm{OH}$ & 830 & $886^{52}$ & $948^{35}$ \\
\hline
\end{tabular}

One can notice that the chlorination of AcAc in $\alpha$-position revealed an opposite effect ${ }^{41,42}$ because of specific steric effects due to the methyl groups.

\section{Structures in vibrational bands of CCC}

As mentioned in the Results section, the spectrum of the chelated enol of 2-CIMA shows some puzzling doublets for several bands. These doublets appear in $\mathrm{pH}_{2}$ and in $\mathrm{Ar}$, but not in Ne. From our previous studies on AcAc in $\mathrm{pH}_{2}$, ${ }^{36}$ we concluded that no site effect was observed under our experimental conditions and a similar conclusion is applied to the study of 2-CIMA in $\mathrm{pH}_{2}$. Anharmonic calculations of 2-CIMA were performed in order to check the possibilities of Fermi or combination bands. A resonance between the intense mode around $1600 \mathrm{~cm}^{-1}$ and a combination mode was found (see Table 2). In fact, the largest and intense doublet in this spectral range (split of $46 \mathrm{~cm}^{-1}$ in $\mathrm{pH}_{2}$ and $22 \mathrm{~cm}^{-1}$ in $\mathrm{Ar}$ ) corresponds to a broad band in $\mathrm{Ne}$, and a vibrational coupling cannot be excluded in this case. Another resonance appeared in the anharmonic approach between the mode around $1345 \mathrm{~cm}^{-1}$ and another combination of modes (see table 2). A resonance between modes, slightly modified by matrix effects, could be at the origin of the weak band detected only in $\mathrm{Ar}$ and $\mathrm{pH}_{2}$ in this range. On the contrary, no resonance occurs in the region of the clearest doublets shown in Figure 3 , eliminating the possibilities of Fermi or combination bands in their case. Moreover, bands coming from these resonances could be shifted in different matrices and show slightly different intensities, but the regular disappearance in $\mathrm{Ne}$ also denies this explanation. These doublets of medium intensity correspond to well defined single bands in $\mathrm{Ne}$. The following discussion will be thus focused on these two specific doublets.

A possible origin of these doublets is the presence of tunnelling splittings due to the hydrogen transfer between the two oxygen atoms as observed in jet experiments with $M A^{15}$. In spite of their absence in the spectrum of MA in matrices, such tunnelling splittings were observed in the IR spectrum of tropolone in Ar and Ne matrices. $^{27}$ As mentioned before, a theoretical estimation of the splitting in the ground state of 2-CIMA gives a value of $12.2 \mathrm{~cm}^{-1}$, compared to $22 \mathrm{~cm}^{-1}$ for MA at the same level of theory. ${ }^{44}$ Considering this value implies that at cryogenic temperature of $3 \mathrm{~K}$, the lowest level is the only one populated in $\mathrm{pH}_{2}$. If the tunnelling splitting is much lower (or possibly reduced by the matrix environment), few wavenumbers or less as in the case of tropolone, both tunnelling levels in the ground state are populated and doublets should come from vibrational transitions towards two well separated tunnelling levels in the vibrationally excited state. In both cases, the observed splitting should reflect the tunnelling in the vibrationally excited level.

The proton tunnelling splitting depends on the vibrational level as observed in the study of tropolone ${ }^{27}$ or in the studies of MA in supersonic jets. ${ }^{13-15}$ The $\mathrm{H}$ transfer requires a rearrangement of the pseudo-cycle favoured in modes implying skeletal deformation. The two well defined doublets correspond to in-plane modes exhibiting these deformations. The $\mathrm{O} \cdots \mathrm{H}$ stretching related to the IHB is involved in both modes, allowing an observable splitting in the excited state. Moreover, from the description of the modes obtained in our calculations, the two modes at play correlate to totally symmetric modes in the G4 molecular symmetry group. Their excitation should increase the tunnelling splitting, following the "rules" discussed in Ref.18 in the case of malonaldehyde. A deeper theoretical investigation would be necessary to predict the effect of the vibrational excitation of the various modes on the tunnelling splitting, but the present basic results seem to favour an important increase of the tunnelling splitting only for these two modes in the $[800-1800 \mathrm{~cm}$ ${ }^{1}$ ] spectral range - almost pure totally symmetric modes in the corresponding $\mathrm{C}_{2 v}$ symmetry of the transient species and $\mathrm{O} \cdots \mathrm{O}$ motion at play -. The tunnelling splitting is reduced in $\mathrm{Ar}$ compared to $\mathrm{pH}_{2}$ (from 8 to 5 $\mathrm{cm}^{-1}$ ) because of a more intrusive $\mathrm{Ar}$ matrix. It means that in any case (one or two populated levels in $\mathrm{pH}_{2}$ ), both ground state levels should be populated at $7 \mathrm{~K}$ in $\mathrm{Ar}$ and that four bands should appear in the spectrum shown in Figure 3a. The observed pattern is effectively more structured in $\mathrm{Ar}$ than in $\mathrm{pH}_{2}$ but it cannot be assigned to the four expected bands if a splitting larger than $3 \mathrm{~cm}^{-1}$ is assumed in the ground level. Beside the isotopic structure, the pattern is more structured in $\mathrm{Ar}$ because of site effects. The attempts of temperature effects in $\mathrm{Ar}$ were not conclusive because of a broadening of the components, even at $15 \mathrm{~K}$, preventing nice quantification of intensity ratios. The Raman spectrum of Figure 4 is not of great help in the assignments of doublets, first because of the slightly higher temperature and second because of the higher concentration, leading to a broadening of the bands. Selection rules are different in IR and in Raman spectra. One can notice than in the case of the doublet around $920 \mathrm{~cm}^{-1}$, the only band observed in Raman corresponds to the less intense in IR, suggesting that the other component is too weak to be detected. In summary, we conclude that the comparison between $\mathrm{pH}_{2}$ and $\mathrm{Ar}$ 
matrices, with components of doublets of comparable intensities in both IR spectra, whereas the spectra are not recorded at the same temperature, is in favour of a weak ground state splitting with population of both levels even in $\mathrm{pH}_{2}$.

The absence of doublets in Ne means that the neon environment prevents $\mathrm{H}$ tunnelling, as suggested in the case of MA in matrices. ${ }^{13}$ No vibrational splitting was found in the case of MA trapped in xenon, neon, argon and nitrogen matrices. ${ }^{24-26}$ The authors concluded that there is a strong environment effect on the tunnelling process. ${ }^{24}$ The quenching of the tunnelling splitting in MA was even observed in Ar coated clusters. However, proton tunnelling splitting in matrices was clearly observed in the case of tropolone ${ }^{27}$, involving a weak tunnelling splitting in the ground state (less than $1 \mathrm{~cm}^{-1}$ in the isolated molecule), ${ }^{53}$ and of 9hydroxyphenalenone $(9-\mathrm{HPO})^{28}$, with a large tunnelling splitting in the ground state $\left(69 \mathrm{~cm}^{-1}\right.$ in Ne). ${ }^{54}$ The matrix induced quenching of the tunnelling splitting can be due (i) to a reduction of the total symmetry in the molecule/matrix system and a breakup of the symmetric double well potential when the molecule is in asymmetric sites, or (ii) to a dynamic friction on the tunnelling coordinate hindering the transfer. The latter effect is preferred in the case of MA. ${ }^{24}$ There is a big difference of molecular size between MA and 9-HPO or tropolone, and thus between their respective trapping sites. The chlorine atom is also rather large and the IHB in 2-CIMA could be more isolated from the matrix atoms than in MA, as suggested in the case of 9-HPO. ${ }^{24}$ These larger molecules are not embedded in the same sites as MA and the coupling with phonons can be weaker. Our experiments on 2-CIMA highlight a dependence on the matrix host. This discussion suggests a stronger interaction between 2-CIMA and the host in Ne than in Ar and $p \mathrm{H}_{2}$, whereas it is well-known that Ne has the weakest effect on the vibrational frequencies because of its lowest polarizability. The observed effect would be then related to a smaller lattice parameter in Ne than in Ar and $p \mathrm{H}_{2}$. In a theoretical study of the proton tunnelling in the isomerization of formic acid embedded in $\mathrm{Ar}, \mathrm{Kr}$ and $\mathrm{Xe}$ matrices, the authors highlight this dependence on the size ratio between the guest and the host: lighter is the noble gas, larger is the rearrangement of the cage. ${ }^{55}$ This study applied to 2CIMA in $\mathrm{Ar}$ and Ne could explain the disappearance of the tunnelling in Ne. We undertook simulations of trapping sites of MA and 2-CIMA in Ne and Ar matrices to get a view on these geometrical questions. The method of the performed classical molecular dynamics is shortly described in ESI. Preliminary results are the following. MA and 2-CIMA are trapped in well-defined crystallographic planes in Ar, removing mainly 3 and $(3$ or 4) Ar atoms respectively, whereas the trapping sites are more chaotic in Ne: 5 or 6 Ne removed for MA and 7 for 2-CIMA with vacancies in more than one crystallographic plane. This difference is in agreement with less molecular perturbations in $\mathrm{Ar}$ than in $\mathrm{Ne}$. Moreover, the Ar lattice seems to leave more place to the proton transfer around 2-CIMA than around MA. Examples of the main trapping sites obtained in $\mathrm{Ar}$ are shown in Figure S1 in ESI. A deeper investigation is necessary to extract valuable conclusions, but these first attempts seem to confirm the role of site geometries on the molecular properties of MA and 2-CIMA.

In conclusion, our data strongly suggest that the welldefined doublets depicted in Figure 3 in $\mathrm{Ar}$ and $\mathrm{pH}_{2}$ can be understood in terms of tunnelling splitting in the excited vibrational levels. Future work included deuteration effects, temperature effects in other matrices is planned. Nevertheless, it is not sure that they will bring valuable information to confirm or infirm the present discussion. On the contrary, our data are fully pertinent for future theoretical investigations on the isolated molecule.

\section{Conclusion}

Matrix isolation of 2-chloromalonaldehyde in different cryogenic solids provides new experimental data on a RAHB system.

The substitution of a hydrogen by a chlorine atom modifies the structure but also the stability of the different conformers. Theoretical results show that the most stable enol conformer is the chelated one (CCC), but experimentally, besides the CCC form, we have detected two other conformers (TTC and CTC) highlighting the presence of a weak bond between the chlorine and the hydrogen of the hydroxyl group. This weak interaction stabilizes these open conformers in the deposited sample. Open isomers are especially studied in a forthcoming publication devoted to UV isomerisation of 2-CIMA in matrices, giving access to their detailed IR spectra.

We show clear experimental evidence that chlorination in the $\alpha$-position in a pseudo-cycle decreases the strength of the IHB. The RAHB system delocalized on the cycle is weakened. This is illustrated by the measured values of the frequencies of the bending out of plane $\mathrm{OH}$ mode in the different matrices. This mode is blue-shifted by the IHB, and the shift is smaller for 2chloromalonaldehyde than for malonaldehyde or acetylacetone.

Nevertheless, the IHB remains strong enough to favor proton transfer, and it could induce a tunnelling splitting in the vibrational levels. We expected to see them in $p \mathrm{H}_{2}$ matrix because this medium preserves large amplitude motions. We observed effectively several doublets which can be the signature of this tunnelling in specific vibrational levels while they were absent in neon matrices. Doublets also exist in argon matrices where substitutional sites are roughly of the same size as in $p \mathrm{H}_{2}$. The splittings are smaller in $\mathrm{Ar}$ than in $p \mathrm{H}_{2}$, in agreement with the softness of $p \mathrm{H}_{2}$.

Complementary experiments with deuterated species would be of great interest, not only for the study of the tunnelling splittings, but also for comparison of the IHB strength with MA through the measure of the $O D$ 
stretching frequency. This band is less broad than the $\mathrm{OH}$ one and has been measured for MA in gas phase. ${ }^{56}$ We could hope to see it in matrices as it was the case for deuterated acetylacetone in $\mathrm{Ne}^{57}$

\section{Conflicts of interest}

There are no conflicts to declare.

\section{Acknowledgments}

We acknowledge the use of the computing center MésoLUM of the LUMAT research federation (FR LUMAT 2764). This work was supported by the RTRA Triangle de la Physique (2010-004-T NOSTADYNE and 2013-0436T REACMAQ) and by the French-Lithuanian PHC GILIBERT program (32894YA).

\section{References}

1 G. Gilli, F. Bellucci, V. Ferretti and V. Bertolasi, Evidence for resonance-assisted hydrogen bonding from crystalstructure correlations on the enol form of the $\beta$-diketone fragment., J. Am. Chem. Soc., 1989, 111, 1023-1028.

2 P. Gilli, L. Pretto and G. Gilli, PA/pKa equalization and the prediction of the hydrogen-bond strength: A synergism of classical thermodynamics and structural crystallography, J. Mol. Struct., 2007, 844-845, 328-339.

3 Y. Zhou, G. Deng, Y.-Z. Zheng, J. Xu, H. Ashraf and Z.-W. Yu Evidences for Cooperative Resonance-Assisted Hydrogen Bonds in Protein Secondary Structure Analogs, Sci. Rep., 2016, 6, 36932

K. T. Mahmudov and A. J. L. Pombeiro, Resonance-Assisted Hydrogen Bonding as a Driving Force in Synthesis and a Synthon in the Design of Materials, Chem. - A Eur. J., 2016, 22, 16356-16398.

Y. H. Mariam and R. N. Musin, Transition from moderate to strong hydrogen bonds: Its identification and physical bases in the case of $\mathrm{O}-\mathrm{H}$ ???O intramolecular hydrogen bonds, J. Phys. Chem. A, 2008, 112, 134-145.

S. SCHEINER, Relationship Between Strength of HydrogenBond and Barrier To Proton-Transfer, Theochem-Journal Mol. Struct., 1993, 113, 65-71.

7 S. L. Baughcum, R. W. Duerst, W. F. Rowe, Z. Smith and E. B. Wilson, Microwave spectroscopic study of malonaldehyde (3-hydroxy-2-propenal). 2. Structure, dipole moment, and tunneling, J. Am. Chem. Soc., 1981, 103, 6296-6303.

S. Baughcum, Z. Smith, E. Wilson and R. Duerst, Microwave spectroscopic study of malonaldehyde. 3. Vibrationrotation interaction and one-dimensional model for proton tunneling ,J. Am. Chem. Soc., 1984, 106, 2260-2265.

C. Duan and D. Luckhaus, High resolution IR-diode laser jet spectroscopy of malonaldehyde, Chem. Phys. Lett., 2004, 391, 129-133.

T. Baba, T. Tanaka, I. Morino, K. M. T. Yamada and K. Tanaka, Detection of the tunneling-rotation transitions of malonaldehyde in the submillimeter-wave region, J. Chem. Phys., 1999, 110, 4131-4133.
C. J. Seliskar and R. E. Hoffmann, On the Infrared Spectrum of Malonaldehyde, A Tunneling Hydrogen-Bonded Molecule, 1982, 155, 146-155.

\section{E. S. Goudreau, The University of New Brunswick, 2017.}

T. N. Wassermann, D. Luckhaus, S. Coussan and M. A. Suhm, Proton tunneling estimates for malonaldehyde vibrations from supersonic jet and matrix quenching experiments., Phys. Chem. Chem. Phys., 2006, 8, 23442348.

N. O. B. Lüttschwager, T. N. Wassermann, S. Coussan and M. A. Suhm, Periodic bond breaking and making in the electronic ground state on a sub-picosecond timescale: $\mathrm{OH}$ bending spectroscopy of malonaldehyde in the frequency domain at low temperature, Phys. Chem. Chem. Phys., $2010,12,8201$

N. O. B. Lüttschwager, T. N. Wassermann, S. Coussan and M. A. Suhm, Vibrational tuning of the Hydrogen transfer in malonaldehyde - a combined FTIR and Raman jet study ${ }^{\dagger}$, Mol. Phys., 2013, 111, 2211-2227.

F. Fillaux and B. Nicolaï, Proton transfer in malonaldehyde: From reaction path to Schrödinger's Cat, Chem. Phys. Lett., 2005, 415, 357-361.

V. A. Benderskii, E. V. Vetoshkin, I. S. Irgibaeva and H. P. Trommsdorff, Tunneling splittings in vibrational spectra of non-rigid molecules: IX. Malonaldehyde and its isotopomers as a test case for fully coupled multidimensional tunneling dynamics, Chem. Phys., 2000, 262, 393-422.

W. Siebrand, Z. Smedarchina and A. Fernández-Ramos, Communication: Selection rules for tunneling splitting of vibrationally excited levels, J. Chem. Phys., DOI:10.1063/1.4813002

Y. Yang and M. Meuwly, A generalized reactive force field for nonlinear hydrogen bonds: Hydrogen dynamics and transfer in malonaldehyde, J. Chem. Phys., DOI:10.1063/1.3447701

M. Schröder, F. Gatti and H. D. Meyer, Theoretical studies of the tunneling splitting of malonaldehyde using the multiconfiguration time-dependent Hartree approach, J. Chem. Phys., 2011, 134, 234307.

T. Hammer, M. D. Coutinho-Neto, A. Viel and U. Manthe, Multiconfigurational time-dependent Hartree calculations for tunneling splittings of vibrational states: Theoretical considerations and application to malonaldehyde, J. Chem Phys., 2009, 131, 224109

T. Hammer and U. Manthe, Iterative diagonalization in the state-averaged multi-configurational time-dependent Hartree approach: Excited state tunneling splittings in malonaldehyde, J. Chem. Phys., 2012, 136, 54105.

F. Wu, Y. Ren and W. Bian, The hydrogen tunneling splitting in malonaldehyde: A full-dimensional time-independent quantum mechanical method, J. Chem. Phys., 2016, 145 74309.

D. W. Firth, P. F. Barbara and H. P. Trommsdorff, Matrix induced localization of proton tunneling in malonaldehyde, Chem. Phys., 1989, 136, 349-360.

T. Chiavassa, P. Verlaque, L. Pizzala, A. Allouche and P. Roubin, Experimental and Theoretical Studies of the Photoisomerization of Malonaldehyde Isolated in Rare Gas Matrices, J. Phys. Chem., 1993, 97, 5917-5925. 

A. Trivella, S. Coussan, T. Chiavassa, P. Theule, P. Roubin and $\mathrm{C}$. Manca, Comparative study of structure and photoinduced reactivity of malonaldehyde and acetylacetone isolated in nitrogen matrices, Low Temp. Phys., 2006, 32, 1042-1049.

R. L. Redington and T. E. Redington, Tropolone monomer Vibrational Spectrum and Proton Tunneling, J. Mol. Spectrosc., 1979, 78, 229-247.

P. F. Barbara, P. K. Walsh and L. E. Brus, Feature article: Picosecond kinetic and vibrationally resolved spectroscopic studies of intramolecular Excited-state Hydrogen Atom Transfer, J. Phys. Chem., 1989, 93, 29-34.

J. N. Woodford, Density functional theory and atoms-inmolecules investigation of intramolecular hydrogen bonding in derivatives of malonaldehyde and implications for resonance-assisted hydrogen bonding, J. Phys. Chem. A, 2007, 111, 8519-8530.

N. Nagashima, S. Kudoh, M. Takayanagi and M. Nakata, UVinduced photoisomerization of acetylacetone and identification of less-stable isomers by low-temperature matrix-isolation infrared spectroscopy and density functional theory calculation, J. Phys. Chem. A, 2001, 105, 10832-10838.

S. Coussan, Y. Ferro, A. Trivella, M. Rajzmann, P. Roubin, R. Wieczorek, C. Manca, P. Piecuch, K. Kowalski, M. Włoch, S. A. Kucharski and M. Musiał, Experimental and theoretical UV characterizations of acetylacetone and its isomers, J. Phys. Chem. A, 2006, 110, 3920-3926.

A. Trivella, T. N. Wassermann, J. M. Mestdagh, C. M Tanner, F. Marinelli, P. Roubin and S. Coussan, New insights into the photodynamics of acetylacetone: isomerization and fragmentation in low-temperature matrixes, Phys. Chem. Chem. Phys., 2010, 12, 8151

S. A. Broadbent, L. A. Burns, C. Chatterjee and P. H. Vaccaro, Investigation of electronic structure and proton transfer in ground state acetylacetone, Chem. Phys. Lett. 2007, 434, 31-37.

A. Trivella, P. Roubin, P. Theule, M. Rajzmann, S. Coussan and $C$. Manca, UV and IR photoisomerization of acetylacetone trapped in a nitrogen matrix, J. Phys. Chem. A, 2007, 111, 3074-3081.

R. R. Lozada-Garcia, J. Ceponkus, W. Chin, M. Chevalier and C. Crépin, Acetylacetone in hydrogen solids: IR signatures of the enol and keto tautomers and UV induced tautomerization, Chem. Phys. Lett., 2011, 504, 142-147.

R. R. Lozada-García, J. Ceponkus, M. Chevalier, W. Chin, J.M. Mestdagh and C. Crépin, Photochemistry of acetylacetone isolated in parahydrogen matrices upon 266 nm irradiation., Phys. Chem. Chem. Phys., 2012, 14, 34503459.

37 R. R. Lozada-Garcia, J. Ceponkus, M. Chevalier, W. Chin, J. M. Mestdagh and C. Crépin, Nuclear spin conversion to probe the methyl rotation effect on hydrogen-bond and vibrational dynamics, Angew. Chemie - Int. Ed., 2012, 51 6947-6950

Y. Wang, B. J. Braams, J. M. Bowman, S. Carter and D. P. Tew, Full-dimensional quantum calculations of groundstate tunneling splitting of malonaldehyde using an accurate ab initio potential energy surface, J. Chem. Phys., 2008, 128, 1-9. and S. F. Tayyari, Structure and vibrational assignment of the enol form of 1,1,1-trifluoro-2,4-pentanedione, Spectrochim. Acta - Part A Mol. Biomol. Spectrosc., 2006, 65, 387-396.

L. Evangelisti, S. Tang, B. Velino, B. M. Giuliano, S. Melandri and W. Caminati, Hexafluoroacetylacetone: A 'rigid' molecule with an enolic Cs shape, Chem. Phys. Lett., 2009 $473,247-250$

A. F. Jalbout, M. Ali Naseri, M. Fazli, H. Raissi, M. Rezaei, A. Nowroozi and A. De Leon, Molecular structure and vibrational assignment of Chloro Acetylacetone: A density fonctional theory study, Int. J. Quantum Chem., 2009, 109, 1481-1496.

S. F. Tayyari, M. Zahedi-Tabrizi, R. Afzali, S. Laleh, H. A Mirshahi and Y. A. Wang, Structure and vibrational assignment of the enol form of 3-chloro-pentane-2,4dione, J. Mol. Struct., 2008, 873, 79-88.

Y. Nishimura, H. Ujita and H. Sekiya, Structures and Proton Transfer of Malonaldehyde, Kasuga, Fukuoka 816, 1993, vol. 15

M. A. Rios and J. Rodriguez, Analysis of the effect of substitution on the intramolecular hydrogen bond of MA by ab initio calculations at the 3-21G level, J. Mol. Struct., 1991, 228, 149-158.

J. C. Hargis, F. A. Evangelista, J. B. Ingels and H. F. S. lii, Short Intramolecular Hydrogen Bonds : Derivatives of Malonaldehyde with Symmetrical Substituents Short Intramolecular Hydrogen Bonds : Derivatives of Malonaldehyde with Symmetrical Substituents, 2008, 17471-17478.

M. Palusiak, S. Simon and M. Solà, The proton transfer reaction in malonaldehyde derivatives: Substituent effects and quasi-aromaticity of the proton bridge, Chem. Phys. 2007, 342, 43-54.

E. Nakhaei and A. Nowroozi, On the performance of resonance assisted hydrogen bond theory in malonaldehyde derivatives, Comput. Theor. Chem., 2016, 1096, 27-32.

A. Nowroozi, H. Raissi, H. Hajiabadi and P. Mohammadzadeh Jahani, Reinvestigation of Intramolecular Hydrogen Bond in Malonaldehyde Derivatives: An Ab initio, AIM and NBO Study, Int. J. Quantum Chem., 2011, 111, 3040-3047.

T. Oka, High-Resolution Spectroscopy of Solid Hydrogen, Annu. Rev. Phys. Chem., 1993, 44, 299-333.

A. Gutiérrez-Quintanilla, M. Chevalier, R. Platakyte, J. Ceponkus and C. Crépin, Prep., 2017.

S. Tam and M. E. Fajardo, Ortho/para hydrogen converter for rapid deposition matrix isolation spectroscopy, Rev. Sci. Instrum., 1999, 70, 1926-1932.

A. Trivella, Université de Provence, 2007.

K. Tanaka, H. Honjo and T. Tanaka, Determination of the proton tunneling splitting of tropolone in the ground state by microwave spectroscopy, J. Chem. Phys., 1999, 110, 1969.

V. E. Bondybey, R. C. Haddon and J. H. English, Fluorescence and phosphorescence of 9hydroxyphenalenone in solid neon and its hydrogen tunneling potential function, J. Chem. Phys., 1984, 80 
$5432-5437$

55

L. I. Trakhtenberg, A. A. Fokeyev, A. S. Zyubin, A. M. Mebel and S. H. Lin, Effect of the medium on intramolecular Hatom tunneling: Cis-trans conversion of formic acid in solid matrixes of noble gases, J. Phys. Chem. B, 2010, 114, 17102-17112.

Z. Smith, E. B. Wilson and R. W. Duerst, The infrared spectrum of gaseous malonaldehyde (3-hydroxy-2propenal), Spectrochim. Acta Part A Mol. Spectrosc., 1983, 39, 1117-1129.

A. Gutierrez-Quintanilla, M. Chevalier and C. Crepin, Double deuterated acetylacetone in neon matrices: infrared spectroscopy\{,\} photoreactivity and the tunneling process, Phys. Chem. Chem. Phys., 2016, 18, 20713-20725. 


\section{Journal Name}

\section{ARTICLE}

